\title{
A Noncommutative Model of Cosmology with Two Metrics
}

\author{
Horacio Falomir ${ }^{1}$, Jorge Gamboa ${ }^{2}$ and Fernando Mendez ${ }^{2, *}$ (D) \\ 1 Departamento de Física, Universidad Nacional de La Plata, La Plata 1900, Argentina; falomir@fisica.unlp.edu.ar \\ 2 Departamento de Física, Universidad de Santiago de Chile, Casilla 307, Santiago 8320000, Chile; \\ jorge.gamboa@usach.cl \\ * Correspondence: fernando.mendez@usach.cl
}

Received: 14 November 2019; Accepted: 30 December 2019; Published: 9 March 2020

\begin{abstract}
We propose a bicosmology model which reduces to the classical analog of noncommutative quantum mechanics. From this point of view, one of the sources in the so modified Friedmann-RobertsonWalker equations is a kind of dark energy governed by a Chapligyn-like equation of state. The parameters of noncommutativity $\theta$ and $B$ are interpreted in terms of the Planck area and a magnetic-like field, which presumably acts as a seed for magnetogenesis.
\end{abstract}

Keywords: noncommutativity; cosmology; dark energy

\section{Introduction}

The possibility that spacetime has an underlying structure at small distance scale-or high energy scale-has been part of long standing research in recent years, mainly driven for the search of a Quantum Gravity Theory [1-8]. Departures from a continuous Minkowski four-dimensional manifold is one possible consequence of this approach, and the loss—or deformation — of Lorentz symmetry at such scales turns out to be an interesting issue.

In this context, the spacetime endowed with a noncommutative structure (Noncommuting coordinates date back to the works of Peierls (Peierls, Z. Phys. 80, 763 (1933)), even if the idea was already suggested by Heisenberg in 1930 (Heisenberg W, Z. Phys. 654 (1930)). An explicitly Lorentz invariant model with noncommutative coordinates was built in 1947 by Snyder (H.S. Snyder Phys. Rev. 71, 38 (1947))) has been a route intensively explored in the last twenty years including noncommutative quantum field theories, noncommutative quantum mechanics, and also noncommutativity in the the space of fields, rather than the usual noncommutative spacetime [9-16].

In this regard, the possibility of non-standard commutation relations or, at the classical level, the introduction of deformed Poisson bracket structures (even in the whole phase space) [17-22] allows for exploring new routes to explain old problems. In this paper, we will explore the implications of such constructions in cosmology, where the observational evidence collected in the last forty years shows not only a remarkable agreement with the standard cosmological model, but also shows that the universe is expanding at an accelerated rate, requiring the incorporation of dark energy in order to explain such acceleration [23].

As much as the standard model of particles needs to be modified in order to incorporate the dark matter [24-30], the existence of dark energy requires ideas beyond the standard cosmological model. In this paper, we will show that the incorporation of a second Friedmann's scale factor together with a non 
standard Poisson structure might shed some light on the origin of dark energy and of magnetic fields in the universe. The mechanisms to originate a magnetic seed might require, in principle, the breaking of a symmetry that is possibly hidden, appearing as an effective degree of freedom-this would be similar to the magnetic field of a magnet that has a purely quantum origin-and the mechanism proposed in the present paper rests on the deformation of the Poisson bracket structure in the space of field.

The assumption about the existence of a second Friedmann's scale factor-which would represent the existence of different patches in the universe, or even other universes, causally disconnected as in bubbles models [31-35]—can not be theoretically ruled out. On the other hand, if the cosmological principle does not reflect an exact symmetry, the transference of information between patches should be a process that would leave some observable traces. This poses the question about the information transfer mechanism.

One of the goals of this letter is to offer an approach that combines the idea of bimetric gravity [36-40] and a relationship with noncommutative quantum mechanics [41] (for a general discussion on bimetric gravity in cosmology, see e.g., [42]).

The idea sketched above allows for incorporating considerations about gauge invariance, causality, and evolution of states, thus providing a different approach to those modern cosmological problems.

This approach has interesting implications because it allows for giving physical interpretations to both infrared and ultraviolet scales.

The paper is organized as follows: in Section 2, we will review some basic aspects of the Friedmann-Robertson-Walker (FRW) metric as well as the extension of the model as it was considered in [43]. Section 3 is devoted to presenting and developing the model in a fully non-commutative phase space. Section 4 provides the conclusions.

\section{Modified FRW Equations}

Let us describe briefly the standard FRW model with one scale factor in order to fix notation. We consider the metric

$$
d s^{2}=-N(t)^{2} d t^{2}+a(t)^{2}\left(\frac{d r^{2}}{1-k r^{2}}+r^{2} d \Omega^{2}\right),
$$

where coordinates are $\{t, r, \theta, \varphi\} ; k=\{-1,0,1\}$ is the spatial curvature and $N(t)$ is the lapse shift.

Einstein's equations for the metric (1) reduce to the FRW equations which, in the gauge $N=1$, turn out to be

$$
\begin{aligned}
2 \frac{\ddot{a}}{a}+\left(\frac{\dot{a}}{a}\right)^{2} & =\Lambda+\cdots, \\
\left(\frac{\dot{a}}{a}\right)^{2} & =\frac{1}{3} \Lambda+\cdots,
\end{aligned}
$$

where $\cdots$ denote contributions from matter fields. From here on, we restrict ourselves to the case $k=0$, which is also consistent with present observations [44] and, for the sake of clarity, we will also omit the matter contributions.

The previous equations can then be obtained from the Lagrangian

$$
L=\frac{1}{2 N} a \dot{a}^{2}+\frac{N}{6} \Lambda a^{3}
$$

Indeed, while Equation (2) results from variations with respect to the variable $a$, the second one (3) results from variations with respect to $N$. 
It will be useful for our purposes to describe this model, through a nonlinear transformation of the dynamical variable, as a harmonic oscillator with imaginary frequency. Indeed, consider the change of variables

$$
x=\frac{2}{3 \sqrt{G}} a^{3 / 2} .
$$

In terms of this new variable, which has dimensions of (energy $)^{-1 / 2}$, the Lagrangian in (4) changes into

$$
G^{3 / 2} L=\frac{1}{2 N} \dot{x}^{2}-\frac{N}{2} \omega^{2} x^{2}
$$

where the frequency is defined as

$$
\omega^{2}=-\frac{3}{4} \Lambda
$$

From here on, we will omit the global factor $G$ in the Lagrangian.

The equations of motion obtained though variations of $L$ in Equation (6) with respect to the variables $x$ and $N$ (in the gauge $N=1$ ) are respectively

$$
\begin{aligned}
\ddot{x}+\omega^{2} x & =0, \\
\dot{x}^{2}+\omega^{2} x^{2} & =0 .
\end{aligned}
$$

Note that the constraint (9) is obtained also from Equation (8) as a first integral. Indeed, multiplying this last equation by $\dot{x}$, we get the equivalent equation

$$
\frac{d}{d t}\left[\dot{x}^{2}+\omega^{2} x^{2}\right]=0
$$

Therefore, the constant $\dot{x}^{2}+\omega^{2} x^{2}$ must be chosen as equal to zero (in which case the energy is zero) and non-trivial solutions are obtained for $\omega$ an imaginary number, as it is upon the identification with the cosmological constant in Equation (7).

Now, we will modify the FRW equations by assuming more than one scale factor. The assumptions of homogeneity and isotropy of each RW patch, together with the assumption of causal disconnection between them, implies that, in the present model, the scale factors $a_{i}(t), i=1,2, \cdots$ satisfy

$$
\left[a_{i}(t), a_{j}(t)\right]=0,
$$

where $[$,$] denotes a Poisson bracket. Note that this "microcausality" principle implies also the possibility$ of gauging the lapse function for all these patches in order to choose one and the same cosmological time for all them.

Interestingly, there is an alternative approach if one assumes the point of view of conformal invariant general relativity theory (We would like to thank the anonymous referee who drew our attention to this subtle issue) $[45,46]$. There, instead of considering two different scale factors, one should introduce two different dilaton fields and the interaction between them will be given —as in the present case-by a deformed Poisson bracket structure. In such case, the so-called unphysical metric (that is, $\bar{g}_{\mu \nu}$ in $\left.d s^{2}=a^{2}(t) \bar{g}_{\mu v} d x^{\mu} d x^{v}\right)$ is chosen as the same for the two patches.

The key observation-in the present model—is to note that, in the harmonic oscillator representation (5), the model admits a straightforward generalization to more than one Friedmann's scale factor.

Let us consider the case of only two patches. Instead of the usual Poisson's bracket between conjugate momenta $p_{i}(t)$, satisfying $\left[p_{i}(t), p_{j}(t)\right]=0$, we will choose $\left[p_{i}(t), p_{j}(t)\right]=F_{i j}$, where $F_{i j}=\epsilon_{i j} B$, with $\epsilon_{i j}$ the antisymmetric symbol and $B$ a constant that can be identified as an effective infrared cut-off. 
The generalization of the Lagrangian $L$ in (6) which incorporates two scale factors and this infrared cut-off is equivalent to a mechanical system defined through the Lagrangian involving two harmonic oscillators,

$$
L_{0}=\frac{1}{2 N}\left(\dot{x}_{1}^{2}+\dot{x}_{2}^{2}\right)-\frac{N}{2}\left(\omega_{1}^{2} x_{1}^{2}+\omega_{2}^{2} x_{2}^{2}\right),
$$

with frequencies

$$
\omega_{1}^{2}=-\frac{3}{4} \Lambda_{1}, \quad \omega_{2}^{2}=-\frac{3}{4} \Lambda_{2}
$$

plus an interaction term

$$
\bar{L}=\frac{B}{2}\left(x_{1} \dot{x}_{2}-x_{2} \dot{x}_{1}\right)
$$

so that the total Lagrangian is given by

$$
L=L_{0}+\bar{L} \text {. }
$$

This Lagrangian formally describes a charged non-relativistic particle moving in a constant magnetic field $B$ pointing in the direction of an $x_{3}$-axis perpendicular to the $\left\langle x_{1}, x_{2}\right\rangle$ plane. This magnetic field is defined in the field space rather than in the spacetime.

The equations of motion (in the gauge $N=1$ ) are now

$$
\begin{aligned}
& \ddot{x}_{1}+\omega_{1}^{2} x_{1}-B \dot{x}_{2}=0, \\
& \ddot{x}_{2}+\omega_{2}^{2} x_{2}+B \dot{x}_{1}=0,
\end{aligned}
$$

while the constraint, a consequence of time reparametrization invariance, reads as

$$
\frac{1}{2}\left(\dot{x}_{1}^{2}+\dot{x}_{2}^{2}\right)+\frac{1}{2}\left(\omega_{1}^{2} x_{1}^{2}+\omega_{2}^{2} x_{2}^{2}\right)=0 .
$$

As in the previous section, condition (16) gives nontrivial solutions for imaginary frequencies, which is just the case at hand.

In order to make contact with the cosmological description, we define, in analogy with Equation (5),

$$
x_{1}=\frac{2}{3 \sqrt{G}} a^{3 / 2}, \quad x_{2}=\frac{2}{3 \sqrt{G}} b^{3 / 2},
$$

and then Equations (15) become

$$
\begin{aligned}
& 2 \frac{\ddot{a}}{a}+\left(\frac{\dot{a}}{a}\right)^{2}=-\frac{4}{3} \omega_{1}^{2}+B \sqrt{a b} \frac{\dot{b}}{a^{2}} \\
& 2 \frac{\ddot{b}}{b}+\left(\frac{\dot{b}}{b}\right)^{2}=-\frac{4}{3} \omega_{2}^{2}-B \sqrt{a b} \frac{\dot{a}}{b^{2}}
\end{aligned}
$$

and the constraint (16) now reads as

$$
\left(\frac{\dot{a}}{a}\right)^{2}=-\left(\frac{2}{3} \omega_{1}\right)^{2}-\frac{1}{a^{3}}\left(\frac{3}{4} \omega_{2}^{2} b^{3}+\dot{b}^{2} b\right) .
$$

The expressions in (18) can be considered as the FRW equations for two patches of the Universe that interact through a constant external like-magnetic field, while (19) is the analog of the equation $G_{00}=-8 \pi G T_{00}$ for the one-metric conventional gravity. 
We would like to emphasize that, in this picture, dark energy emerges as a consequence of the incorporation of a sort of interaction—in the space of gravitational fields—between neighboring patches in spacetime, and these extra contributions add to the cosmological constant $\Lambda_{1}$ and $\Lambda_{2}$, on each patch.

From the point of view of the energy and momentum content in Sector 1, Equations (18) and (19) turn out to be

$$
T_{11}=T_{22}=T_{33}=-B \sqrt{a b} \frac{\dot{b}}{a^{2}},
$$

and

$$
T_{00}=-\left(\frac{b}{a}\right)^{3}\left[\left(\frac{\dot{b}}{b}\right)^{2}+\left(\frac{2}{3} \omega_{2}\right)^{2}\right]-\left(\frac{2}{3} \omega_{1}\right)^{2} .
$$

From these results, one has the following equation of state:

$$
\rho_{b}+\frac{6 \pi}{B^{2}} p_{b}^{2}=\frac{\Lambda_{2}}{8 \pi G}\left(\frac{b}{a}\right)^{3}
$$

which describes a kind of Chapligyn gas. This gas has been discussed extensively in cosmology in connection with the dark energy problem [47-50].

As a final remark, note that the previous model has a straightforward description in terms of a Hamiltonian system with modified Poisson Brackets. Indeed, the mechanical system described by the Lagrangian in (14) can also be described by the Hamiltonian

$$
H=\frac{N}{2}\left(p_{1}^{2}+p_{2}^{2}+\omega_{1}^{2} x_{1}^{2}+\omega_{2}^{2} x_{2}^{2}\right)
$$

with the Poisson's bracket algebra

$$
\left[x_{i}, x_{j}\right]=0, \quad\left[x_{i}, p_{j}\right]=\delta_{i j}, \quad\left[p_{i}, p_{j}\right]=\epsilon_{i j} B .
$$

Note that $x$ has dimensions of (energy) ${ }^{-1 / 2}$ while $p$ has its inverse dimensions. Then, $B$ has dimensions of (energy) $)^{+1}$ and, therefore, the magnetic-like field is $B / \sqrt{G}$.

\section{Noncommutative Phase Space Classical Cosmology}

We observe that it is still possible to consider a more general deformation of the Poisson's algebra by taking a full noncommutative phase space. That is, the introduction of a noncommutative parameter in the bracket between coordinates.

Two energy scales are then present in this scenario, namely the Planck energy $E_{P}=G^{-\frac{1}{2}}$ and the "magnetic energy" $B^{\frac{1}{2}}$ (or, equivalently, the Planck and magnetic lengths), defined through the modified Poisson bracket structure.

In order to explore this system, we restrict ourselves to the case with $\omega_{1}=\omega_{2} \equiv \omega$ and rewrite (21) as

$$
H=N \mathcal{H},
$$

where the constraint reads now

$$
\mathcal{H}=\frac{\omega}{2}\left(\bar{p}_{1}^{2}+\bar{p}_{2}^{2}+\bar{x}_{1}^{2}+\bar{x}_{2}^{2}\right),
$$

with original phase space variables $\left\{x_{i}, p_{j}\right\}$ rescaled according to $x_{i} \rightarrow \bar{x}_{i}=\sqrt{\omega} x_{i}=\bar{x}$ and $p_{j} \rightarrow \bar{p}_{j}=$ $p_{j} / \sqrt{\omega}$. 
The modified Poisson brackets structure, in view of our previous discussion is

$$
\begin{aligned}
{\left[\bar{x}_{i}, \bar{x}_{j}\right] } & =\epsilon_{i j} \bar{G}, \\
{\left[\bar{x}_{i}, \bar{p}_{j}\right] } & =\delta_{i j}, \\
{\left[\bar{p}_{i}, \bar{p}_{j}\right] } & =\epsilon_{i j} \bar{B},
\end{aligned}
$$

where $\bar{G}$ and $\bar{B}$ are the rescaled Newton's constant and magnetic-like seed, respectively, according to $\bar{G}=\sqrt{G} \omega$ and $\bar{B}=\frac{B}{\omega}=\sqrt{G} B_{\text {seed }} / \omega$ with $B_{\text {seed }}=B / \sqrt{G}$

The equations of motion for the variables $\bar{x}_{i}$ turn out to be

$$
\ddot{\bar{x}}_{i}+\Omega^{2} \bar{x}_{i}-\mathcal{B} \epsilon_{i j} \dot{\bar{x}}_{j}=0,
$$

where

$$
\begin{aligned}
\Omega^{2} & =\omega^{2}(1-\bar{G} \bar{B})=\omega^{2}\left(1-G B_{\text {seed }}\right) \\
\mathcal{B} & =\omega(\bar{B}+\bar{G})=\sqrt{G}\left(B_{\text {seed }}+\omega^{2}\right) .
\end{aligned}
$$

Comparing Equations (28) with (15) (with $\omega_{1}=\omega_{2}=\omega$ for the last case), we see that an effective magnetic-like background field, given by

$$
\mathcal{B} / \sqrt{G}=B_{\text {seed }}+\omega^{2},
$$

is generated.

While $\Omega^{2}$ can be seen as an effective cosmological constant, the new contribution $\mathcal{B} / \sqrt{G}$ might be considered as an effective magnetic seed in the universe, even though it will be necessary to incorporate matter in this model in order to see how this new term affects the dynamics of charged particles, for example.

Note that, as in noncommutative quantum mechanics [51-53], there are two phases with $G \mathcal{B}>1$ and $G \mathcal{B}<1$, respectively, separated by a critical point at $G \mathcal{B}=1$.

If we demand $G B_{\text {seed }} \ll 1$, then

$$
B_{\text {seed }} \approx \mathcal{B}_{\text {eff }}+\frac{3}{4} \Lambda
$$

This formula provides a simple and direct link between the magnetogenesis [54,55] and the cosmological constant problem.

Note that the modified Poisson brackets in (25)-(27) can be mapped onto a canonical form under a non-canonical change of variables $\left\{\bar{x}_{i}, \bar{p}_{j}\right\} \rightarrow\left\{X_{i}, P_{j}\right\}$, where the new Hamiltonian is still diagonal [51,53]. Thus, $\mathcal{H}$ can be written as

$$
\frac{H}{N}=\frac{1}{2}\left(P_{1}^{2}+\Omega_{+}^{2} X_{1}^{2}\right)+\frac{1}{2}\left(P_{2}^{2}+\Omega_{-}^{2} X_{2}^{2}\right),
$$

where variables $\left\{X_{1}, P_{1}\right\}$ and $\left\{X_{2}, P_{2}\right\}$ are canonically conjugated, while the variables of the sector labeled as ' 1 ' have zero Poisson bracket with those of sector ' 2 '. The frequencies in $(32)$ are $[51,53]$

$$
\begin{aligned}
\Omega_{ \pm} & = \pm \omega\left[\sqrt{1+\frac{1}{4}(\bar{B}-\bar{G})^{2}} \mp \frac{1}{2}(\bar{B}+\bar{G})\right] \\
& \approx \pm\left[\sqrt{\omega^{2}+\left(\frac{\sqrt{G} \mathcal{B}_{\text {eff }}}{2}\right)^{2}} \mp \frac{\sqrt{G} \mathcal{B}_{\text {eff }}}{2}\right]
\end{aligned}
$$


where, in the last line, we used the fact that $B_{\text {seed }}-\omega^{2}=\mathcal{B}_{\text {eff }}-2 \omega^{2} \approx \mathcal{B}_{\text {eff }}$ when $\omega^{2} \approx-3 \Lambda / 4=\Omega^{2}$. With the same approximations, we finally obtain $\Omega_{ \pm}^{2} \approx \omega^{2}$.

\section{Conclusions}

In this work, we have presented a model of cosmology with two metrics in a full non-commutative phase space. We have shown that, besides the appearance of a term acting like dark energy, there is also a term that could be interpreted as a source of magnetic field. This interpretation is based in the analogy with the description of particles in the presence of a static, external, magnetic field, but, in the present model, it must be taken cautiously since this analogy occurs in the gravitational field space rather than the spacetime. Future investigation must be done, in particular, for the study of charged particles evolving in this model of universe, a problem that will be considered in future works.

We should observe also that there are differences between the quantum Hall effect approach and the magnetogenesis as discussed in [43,54,55]. Indeed, as argued in [43], the causal connection between two spacetime regions takes place in the presence of an external constant magnetic-like field (at first sight presumably from the formation of some galactic halo). This external magnetic-like field would be very small, but not necessarily a magnetic seed. The magnetic seed would be created much earlier, as can be seen from the magnetic displacement (30).

However, this posses some intriguing questions: Is $\mathcal{B}$ a real magnetic field?, What is the mechanism responsible for the creation of this $\mathcal{B}$ ? At first glance, it is interesting to think that the origin of this field could be similar to that of the magnetic field produced by a magnet and, therefore, of purely quantum origin.

Author Contributions: All authors contribute the same to this article. All authors have read and agreed to the published version of the manuscript.

Funding: This work was supported by Dicyt 041831GR (J.G) and 041931MF (F. M.). H.F. thanks ANPCyT, CONICET and UNLP, Argentina, for partial support through grants PICT-2014-2304, PIP 688 and Proy. Nro. 11/X748, respectively.

Acknowledgments: We would like to thank Manu Paranjape and Alexios Polychronakos for enlightening discussions. One of us (J.G.) thanks the DESY theory group and the Alexander von Humboldt Foundation for the hospitality and support.

Conflicts of Interest: The authors declare no conflict of interest.

\section{References}

1. Arzano, M.; Kowalski-Glikman, J. Non-commutative fields and the short-scale structure of spacetime. Phys. Lett. B 2017, 771, 222. [CrossRef]

2. Modesto, L. Fractal Structure of Loop Quantum Gravity. Class. Quant. Grav. 2009, 26, 242002. [CrossRef]

3. Letizia, M.; Liberati, S. Deformed relativity symmetries and the local structure of spacetime. Phys. Rev. D 2017, 95, 046007. [CrossRef]

4. Carlip, S. The Small Scale Structure of Spacetime. arXiv 2009, arXiv:1009.1136.

5. Hossenfelder, S. Minimal Length Scale Scenarios for Quantum Gravity. Living Rev. Rel. 2013, 16, 2. [CrossRef] [PubMed]

6. Rovelli, C.; Smolin, L. Discreteness of area and volume in quantum gravity. Nucl. Phys. B 1995, 442, 593. Erratum in 1995, 456, 753. [CrossRef]

7. Bonder, Y.; Sudarsky, D. Unambiguous Quantum Gravity Phenomenology Respecting Lorentz Symmetry. Rept. Math. Phys. 2009, 64, 169. [CrossRef]

8. Aguilar, P.; Sudarsky, D.; Bonder, Y. Experimental search for a Lorentz invariant spacetime granularity: Possibilities and bounds. Phys. Rev. D 2013, 87, 064007. [CrossRef]

9. Seiberg, N.; Witten, E. String theory and noncommutative geometry. JHEP 1999, 9, 3. [CrossRef]

10. Douglas, M.; Nekrasov, N. Noncommutative field theory. Rev. Mod. Phys. 2001, 73, 977. [CrossRef] 
11. Seiberg, N.; Susskind, L.; Toumbas, N. Space-time noncommutativity and causality. JHEP 2000, 6, 44. [CrossRef]

12. Moffat, J.W. Noncommutative quantum gravity. Phys. Lett. B 2000, 491, 345. [CrossRef]

13. Szabo, R.J. Quantum field theory on noncommutative spaces. Phys. Rep. 2003, 378, 207. [CrossRef]

14. Dunne, G.V.; Jackiw, R.; Trugenberger, C. “Topological” (Chern-Simons) quantum mechanics. Phys. Rev. D 1990, 41, 661. [CrossRef] [PubMed]

15. Mezincescu, L. Star operation in quantum mechanics. arXiv 2000, arXiv:hep-th/0007046.

16. Gamboa, J.; Loewe, M.; Rojas, J.C. Noncommutative quantum mechanics. Phys. Rev. D 2001, 64, 067901. [CrossRef]

17. Bayern, F.; Flato, M.; Frönsdal, C.; Lichnerowicz, A.; Sternheimer, D. Deformation theory and quantization. I. Deformations of symplectic structures. Ann. Phys. 1978, 111, 61.

18. Cattaneo, A.S.; Indelicato, D. Formality and Star Products in Poisson Geometry. In Deformations Quantization and Group Representation; Gutt, S., Rawnsley, J.H., Sternheimer, D., Eds.; Cambridge University Press: Cambridge, UK, 2004.

19. De Wilde, M.; Lecomte, P.B.A. Existence of star-products and of formal deformations of the Poisson Lie algebra of arbitrary symplectic manifolds. Lett. Math. Phys. 1994, 7, 487. [CrossRef]

20. Fedosov, B.V. A simple geometrical construction of deformation quantization. J. Diff. Geom. 1994, 40, 213. [CrossRef]

21. Flato, M.; Lichnerowicz, A.; Sternheimer, D. Crochet de Moyal-Vey et quantification. Sci. Paris Ser. A-B 1976, 283, 19.

22. Kontsevitch, M. Deformation Quantization of Poisson Manifolds. Lett. Math. Phys. 2003, 66, 157. [CrossRef]

23. Joyce, A.; Jain, B.; Khoury, J.; Trodden, M. Beyond the Cosmological Standard Model. Phys. Rept. $2015,568,1$. [CrossRef]

24. Debono, I.; Smoot, G.F. General Relativity and Cosmology: Unsolved Questions and Future Directions. Universe 2016, 2, 23. [CrossRef]

25. Frieman, J.; Turner, M.; Huterer, D. Dark Energy and the Accelerating Universe. Ann. Rev. Astron. Astrophys. 2008, 46, 385. [CrossRef]

26. Mota, D.F.; Shaw, D.J. Evading Equivalence Principle Violations, Cosmological and other Experimental Constraints in Scalar Field Theories with a Strong Coupling to Matter. Phys. Rev. D 2007, 75, 063501. [CrossRef]

27. Hansen, F.K.; Banday, A.J.; Gorski, K.M. Testing the cosmological principle of isotropy: Local power spectrum estimates of the WMAP data. Mon. Not. R. Astron. Soc. 2004, 354, 641. [CrossRef]

28. Bousso, R.; Harnik, R.; Kribs, G.D.; Perez, G. Predicting the Cosmological Constant from the Causal Entropic Principle. Phys. Rev. D 2007, 76, 043513. [CrossRef]

29. Ade, P.A.R.; Aghanim, N.; Arnaud, M.; Arroja, F.; Ashdown, M.; Aumont, J.; Baccigalupi, C.; Ballardini, M.; Banday, A.J.; Barreiro, R.B.; et al. Planck 2015 results-XX. Constraints on inflation. Astron. Astrophys. 2016, 594, A20.

30. Ade, P.A.R.; Aghanim, N.; Arnaud, M.; Arroja, F.; Ashdown, M.; Aumont, J.; Baccigalupi, C.; Ballardini, M.; Banday, A.J.; Barreiro, R.B.; et al. [BICEP2/Keck and Planck Collaborations]. Joint Analysis of BICEP2/Keck Array and Planck Data. Phys. Rev. Lett. 2015, 114, 101301. [CrossRef]

31. Kleban, M. Cosmic Bubble Collisions. Class. Quant. Grav. 2011, 28, 204008. [CrossRef]

32. Gobbetti, R.; Kleban, M. Analyzing cosmic bubble collisions. JCAP 2012, 2012, 25. [CrossRef]

33. Lehners, J.L. Cosmic bounces and cyclic universes. Class. Quant. Grav. 2011, 28, 204004. [CrossRef]

34. Aguirre, A.; Johnson, M.C.; Shomer, A. Towards observable signatures of other bubble universes. Phys. Rev. D 2007, 76, 063509. [CrossRef]

35. Chang, S.; Kleban, M.; Levi, T.S. When worlds collide. JCAP 2008, 804, 34. [CrossRef]

36. Akrami, Y.; Hassan, S.F.; Könnig, F.; Schmidt-May, A.; Solomon, A.R. Bimetric gravity is cosmologically viable. Phys. Lett. B 2015, 748, 37. [CrossRef]

37. Akrami, Y.; Koivisto, T.S.; Mota, D.F.; Sandstad, M. Bimetric gravity doubly coupled to matter: Theory and cosmological implications. JCAP 2013, 1310, 46. [CrossRef]

38. Cusin, G.; Durrer, R.; Guarato, P.; Motta, M. Gravitational waves in bigravity cosmology. JCAP 2015, $1505,30$. [CrossRef] 
39. Deser, S.; Izumi, K.; Ong, Y.C.; Waldron, A. Problems of massive gravities. Mod. Phys. Lett. A 2015, $30,1540006$. [CrossRef]

40. De Rham, C.; Heisenberg, L.; Ribeiro, R.H. On couplings to matter in massive (bi-)gravity. Class. Quant. Grav. 2015, 32, 035022. [CrossRef]

41. Falomir, H.; Pisani, P.A.G.; Vega, F.; Cárcamo, D.; Méndez, F.; Loewe, M. On the algebraic structure of rotationally invariant two-dimensional Hamiltonians on the noncommutative phase space. J. Phys. A Math. Theor. 2016, 49, 055202. [CrossRef]

42. Bamba, K.; Makarenko, A.N.; Myagky, A.N.; Nojiri, S.I.; Odintsov, S.D. Bounce cosmology from $F(R)$ gravity and $F(R)$ bigravity. JCAP 2014, 1401, 8. [CrossRef]

43. Falomir, H.; Gamboa, J.; Mendez, F.; Gondolo, P. Magnetic seed and cosmology as quantum hall effect. Phys. Lett. B 2018, 785, 399. [CrossRef]

44. Tanabashi, M; et al. [Particle Data Group]. The Review of Particle Physics. Phys. Rev. D 2018, 98, 030001. [CrossRef]

45. 't Hooft, G. Singularities, Horizons, Firewalls, and Local Conformal Symmetry. In 2nd Karl Schwarzschild Meeting on Gravitational Physics; Nicolini, P., Kaminski, M., Mureika, J., Bleicher, M., Eds.; Springer Proceedings in Physics; Springer: Cham, Swizerland, 2018; Volume 208.

46. 't Hooft, G. A class of elementary particle models without any adjustable real parameters. Found. Phys. 2011, 41, 1829. [CrossRef]

47. Gorini, V.; Kamenshchik, A.; Moschella, U. Can the Chaplygin gas be a plausible model for dark energy? Phys. Rev. D 2003, 67, 063509. [CrossRef]

48. Gorini, V.; Kamenshchik, A.; Moschella, U.; Pasquier, V. The Chaplygin gas as a model for dark energy. AIP Conf. Proc. 2005, 751, 108.

49. Gorini, V.; Kamenshchik, A.Y.; Moschella, U.; Piattella, O.F.; Starobinsky, A.A. Gauge-invariant analysis of perturbations in Chaplygin gas unified models of dark matter and dark energy. JCAP 2008, 802, 16. [CrossRef]

50. Melchiorri, A.; Mersini, L.; Ödman, C.J.; Trodden, M. The State of the dark energy equation of state. Phys. Rev. D 2003, 68, 043509. [CrossRef]

51. Nair, V.P.; Polychronakos, A.P. Quantum mechanics on the noncommutative plane and sphere. Phys. Lett. B 2001, 505, 267. [CrossRef]

52. Bellucci, S.; Nersessian, A.; Sochichiu, C. Two phases of the noncommutative quantum mechanics. Phys. Lett. B 2001, 522, 345. [CrossRef]

53. Carmona, J.M.; Cortes, J.L.; Gamboa, J.; Méndez, F. Quantum theory of noncommutative fields. JHEP 2003, $303,58$. [CrossRef]

54. Kandus, A.; Kunze, K.E.; Tsagas, C.G. Primordial magnetogenesis. Phys. Rept. 2011, 505, 1. [CrossRef]

55. Ratra, B. Cosmological 'seed' magnetic field from inflation. Astrophys. J. 1992, 391, L1. [CrossRef]

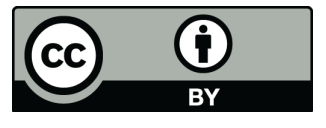

(C) 2020 by the authors. Licensee MDPI, Basel, Switzerland. This article is an open access article distributed under the terms and conditions of the Creative Commons Attribution (CC BY) license (http:/ / creativecommons.org/licenses/by/4.0/). 